\title{
Experimental Analysis of RSSI-Based Indoor Location Systems with WLAN Circularly Polarized Antennas
}

\author{
P. Nepa ${ }^{1}$, F. Cavallo ${ }^{2}$, M. Bonaccorsi ${ }^{2}$, M. Aquilano ${ }^{2}$, \\ M.C. Carrozza ${ }^{2}$, and P. Dario ${ }^{2}$ \\ ${ }^{1}$ Dept. of Information Engineering, University of Pisa, \\ Via G. Caruso 16, I-56122 Pisa, Italy \\ p.nepa@iet.unipi.it \\ ${ }^{2}$ ARTS Lab, Scuola Superiore S. Anna \\ Pisa, Italy \\ \{f.cavallo,m.bonaccorsi,m.acquilano,m.carrozza, \\ p.dario\} @sssup.it
}

\begin{abstract}
Circularly polarized antennas are used in $2.4 \mathrm{GHz}$ ZigBee radio modules to evaluate performance improvement of RSSI (Received Signal Strength Indicator) based location techniques, with respect to conventional linearly polarized antennas. Experimental RSSI measurements in an indoor environment clearly show that multipath fading is significantly reduced when $\mathrm{CP}$ antennas are used; this determines a more reliable estimation of the field amplitude decay law as a function of the distance of the mobile node from the fixed access point, and then a higher location accuracy. At the best of authors' knowledge, it is the first time that the circular polarization features are applied to RSSI-based radio location techniques.
\end{abstract}

Keywords: indoor location, RSSI-based location, circularly polarized antennas.

\section{Introduction}

In microwave radio links, the effectiveness of the circular polarization $(\mathrm{CP})$ in reducing multipath effects is well known; $\mathrm{CP}$ also allows more flexible reciprocal orientation of the transmitter and the receiver antenna. For the above reasons, circularly polarized antennas are used in a number of wireless systems, as for example the GPS (Global Positioning System) system and satellite-to-mobile wideband communication links, as well as most UHF RFID (Radio Frequency IDentification) readers. Moreover, several ATC (Air Traffic Control) radars and SAR (Synthetic Aperture Radar) systems adopt $\mathrm{CP}$ antennas, by bringing into play some interesting properties of the polarimetric scattering.

When a circularly polarized wave is incident on a reflecting surface at a small incident angle (near to the normal incidence condition), the handedness of the circular polarization of the reflected wave is reversed: if a right (left) hand CP signal is transmitted, it would become a left (right) hand CP signal after its first reflection; after the second reflection, it would again become a right (left) hand CP signal, and so on. Thus, if the transmitting antenna is circularly polarized and the receiving antenna polarization is also circular with the same handedness as the transmitting antenna, 
multipath delayed waves after single reflection will be effectively rejected on reception by the receiving antenna. Indeed, it has been shown [1]-[2] that the RMS delay spread for circular polarization is about half of that for linear polarization (in both cases, the same polarization was used for the transmitter and the receiver).

In wireless local area networks (WLANs), circular polarized antennas are suggested for the access points, since a reliable radio links can be obtained independently on the spatial orientation of the mobile node antenna (which often is a linearly polarized antenna).

In the open literature, a comparative analysis between vertical and horizontal linear polarization antennas has been performed, in ZigBee WSNs for localization purposes [3].

Although the superiority of circular polarization over linear polarization for the suppression of the effects of multipath propagation in radio channels is well known, to the best of the authors' knowledge, there has not been a deep investigation on the improvement that can be achieved when $\mathrm{CP}$ antennas are used for the fixed/mobile nodes of wireless sensor networks (WSNs), in the context of RSSI (Received Signal Strength Indicator) based indoor location systems. In indoor environments, the performance of RSSI-based location systems is significantly reduced due to the multipath phenomena which determines rapid spatial variations of the electromagnetic field amplitude [4,5]: significant amplitude variations are obtained in short distances [6], which is apparently related to the relatively short wavelength (around $12.5 \mathrm{~cm}$ a $2.4 \mathrm{GHz}$ ).

It is worth noting that in indoor location systems multipath and Non Line Of Sight (NLOS) conditions make range-free localization methods (like fingerprinting) more reliable than range-based ones [7,8]. Therefore, fading attenuation through the adoption $\mathrm{CP}$ antennas can be an effective technique to enhance performance and competitiveness of range-based methods. Furthermore, it should be underlined that the adoption of CP antennas in WLANs, instead of more standard LP antennas, determines a negligible influence on cost and performance of the wireless communication system.

In this paper, preliminary location accuracy measurements for an RSSI-based location technique are shown, when circularly polarized antennas are used at both the anchors and the mobile node of a WSN, and compared with those obtained when conventional linearly polarized antennas are adopted [9]. Measurements with CP antennas have been performed in the same scenario as that for the measurements with conventional linear polarization antennas.

\section{Measurement Set-Up}

A ZigBee-based wireless sensor network was set-up to measure RSSI over distance in a typical indoor environment and perform a simple localization task in 1-D. The network is composed of ZigBee boards (EM250 from Ember) and is conceived to have three typologies of nodes: coordinator node, mobile node (MN) and anchor node. The coordinator node is USB-connected to a personal computer and is used to create and hold the network, acquire and process data (a graphical interface developed in $\mathrm{C \#}$ is used for network management and data saving); the MN periodically send messages to anchors to allow for RSSI measurements at each fixed node. Anchors are placed at fixed and known positions, measure the RSSI value and transmit the recorded value to the coordinator node. 
In a preliminary experimental set-up, two anchors were placed tree meters apart and a MN mounted on a straightforward aluminium rail has been remotely moved between anchors. A plastic support mounted on the aluminium rail is used to keep nodes $90 \mathrm{~cm}$ above the ground (see Fig. 1).

Two types of antennas have been used, to evaluate advantages of using Circular Polarization (CP) instead of Linear Polarization (LP) in RSSI-based indoor location. Murata antennas (ceramic antennas) embedded in EM250 modules were used as LP antennas, while external patch antennas from Hyperlink Technologies (HG2409PCR) were used as CP antennas. The patch antenna is connected to EM250 by means of an MC-CARD external antenna connector and an MC-CARD-to-12-in-N-FEMALE adaptor cable. When the external patch antenna is connected to the EM250 module via MC-CARD connector, an internal switch automatically excludes native Murata antenna from radio module and only the external antenna can radiate. For both polarization cases, transmitting and receiving antennas face each other along their maximum radiation direction, and are at the same height from the floor (they don't rotate during MN translation).

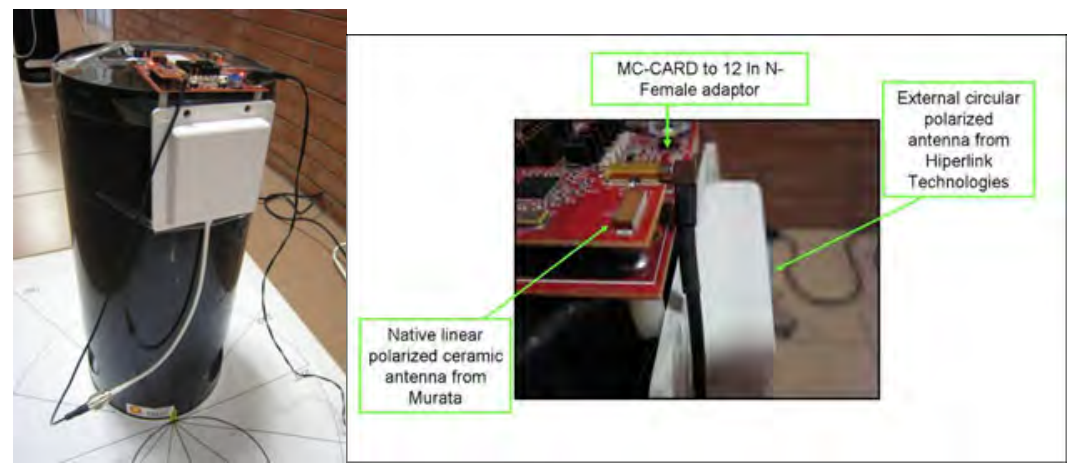

Fig. 1. The plastic support for the wireless node, where the radio module (on the left) and both the ceramic and patch antennas (on the right) are visible

Measurements were conducted in a repository of ARTS Laboratory. In order to perform RSSI characterization with an adequate spatial resolution, a commercial motion capture system was used, also to guarantee experiment repeatability. A six-camera Vicon 460 system has been installed to capture MN positions. Vicon is a motion capture device based on infrared cameras and IR refractive markers to be attached to the tracked objects. The system is composed of:

1. Six IR digital cameras: cameras are wire connected to Vicon computer workstation, each camera has an autofocus lens (from Sigma) and a ring IR-LED headlight for workspace IR spotting;

2. IR reflective markers: Vicon system can recognize markers thanks to their IR reflectivity and standard dimensions; markers must be attached to the objects for their localization and tracking, and must be visible from at least three cameras simultaneously; 
3. An ad-hoc computer station for system calibration, marker tracking, data management and processing;

4. Connection cables and camera/computer interfaces unit for data transfer and cameras power supply.

Stereoscopic vision from at least three cameras is performed to reconstruct 3-D marker location and motion. Every Vicon's camera is on a stable and fixed easel. They spot a piece or the entire workspace in IR bandwidth and illuminate the scene using IR leds. Passive reflective IR markers mounted on monitored objects appear as white spots to the camera. A specific software for marker recognition allows for automatic marker labelling and tracking. A calibration procedure is needed in order to create a common global reference system for all involved cameras and compute the 3-D position of IR markers.

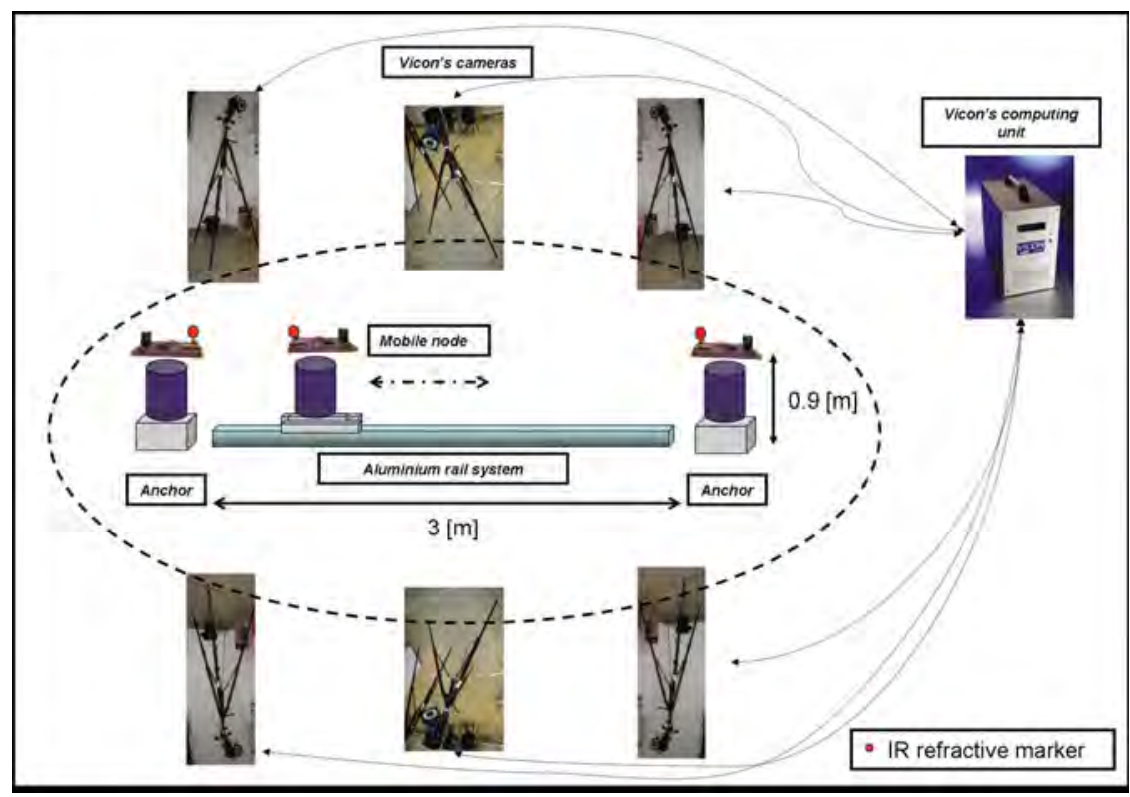

Fig. 2. A schematic description of the whole system made of six IR cameras, two anchors (actually only one has been used to get preliminary results) and a mobile node moving along an aluminium rail

Calibration procedure consists of two steps, a static and a dynamic one:

- Static calibration:

Static calibration procedure create a prospective projection matrix for each camera to relate camera image reference system coordinate (2-D) with global reference system coordinate (3-D).

- Dynamic calibration:

Dynamic calibration perform error assessment in IR TAG localization during marker motion along workspace. If large errors occur a new calibration 
procedure can be done, otherwise, calibration errors are computed and system is ready for marker tracking task.

After calibration, Vicon is ready for marker localization and tracking. Standard markers are fixed on antennas to reconstruct antenna position and motion during MN translation. When Vicon acquisition procedure starts, 3-D coordinate are continuously recorded on a txt file. At the end of the acquisition, 3-D trajectories are reconstructed and data can be used for further elaborations. Markers can be manually or automatically labelled in 3-D reconstructed vision to distinguish antennas and save relative position coordinates on a txt file. A system accuracy of less than $1 \mathrm{~mm}$ can be achieved after calibration procedure. To achieve a satisfactory experiment reliability, the $\mathrm{MN}$ was fixed at an aluminium rail and remotely moved by a wire. The MN moved from about $6 \mathrm{~cm}$ to $275 \mathrm{~cm}$ from the anchor during the experimental trial, and speed was maintained as constant as possible to get an uniform spatial sampling rate. $\mathrm{MN}$ and anchors were fixed to allow only a translation along rail axis, avoiding any rotation.

MN continuously sent data to the anchors during on-rail translation at a frequency of $10 \mathrm{~Hz}$ and Vicon capture device synchronously computed and recorded IR TAG position at a sampling rate of $100 \mathrm{~Hz}$ (data position acquired by means of the Vicon system were decimated at a $10 \mathrm{~Hz}$ frequency in order to be conformed to the RSSI sampling rate). A mean spatial sampling of $1.2 \mathrm{~cm}$ has been reached during experimental trials. Post process of the recorded RSSI-values by using Matlab gave us RSSI spatial characterization. Anchors-MN relative positions and distances were reconstructed thanks to Vicon's data, and used as reference data.

\section{Experimental Results}

Samples of RSSI traces are given in Fig. 3, for both polarization cases, as a function of the distance between an anchor and the mobile node moving along the aluminium rail. For each polarization case, measurements have been repeated 10 times; the ten curves are almost overlapped, so confirming the reliability of the measurement set-up. When LP antennas are used, the fading generated from the interference between the direct ray and those reflected from walls, ceiling and floor is apparent. Constructive and destructive interferences due to superposition of incident and reflected waves in the environment produce relatively high RSSI spatial fluctuations. On the other hand, reduced oscillations can be observed when $\mathrm{CP}$ antennas are used, as expected due to the reduced amplitude of the first order reflections with the same $\mathrm{CP}$ handedness as that of the direct ray. A higher amplitude of the RSSI values for CP polarization is related to the higher gain of the $\mathrm{CP}$ antennas $(8 \mathrm{dBi}$ instead of the $0 \mathrm{dBi}$ of the ceramic LP antennas). Small fluctuations are still present in RSSI traces for CP polarization. Indeed, multipath delayed waves that have undergone single reflection are not always suppressed effectively due to the fact that the antenna axial ratio, AR, is not ideal $(A R>0 d B)$, and it changes in the antenna main beam and impedance bandwidth (typical values are $A R<2-3 \mathrm{~dB}$ ). Moreover, far from the normal incidence direction the reflection of a $\mathrm{CP}$ signal from a wall or ceiling (which are not perfectly conducting surfaces) gives rise to an elliptically polarized signal rather than a $\mathrm{CP}$ signal (with reversed handedness). The latter phenomenon is more evident at grazing incidence. Moreover, it must be considered that in indoor environments higher-order reflections 
are always present. Nevertheless, the use of circular polarization can still be effective, by considering the fact that the channel fading is primarily caused by first-order reflections, and the field amplitude of singly reflected waves is generally much higher than that of higher-order reflection waves.

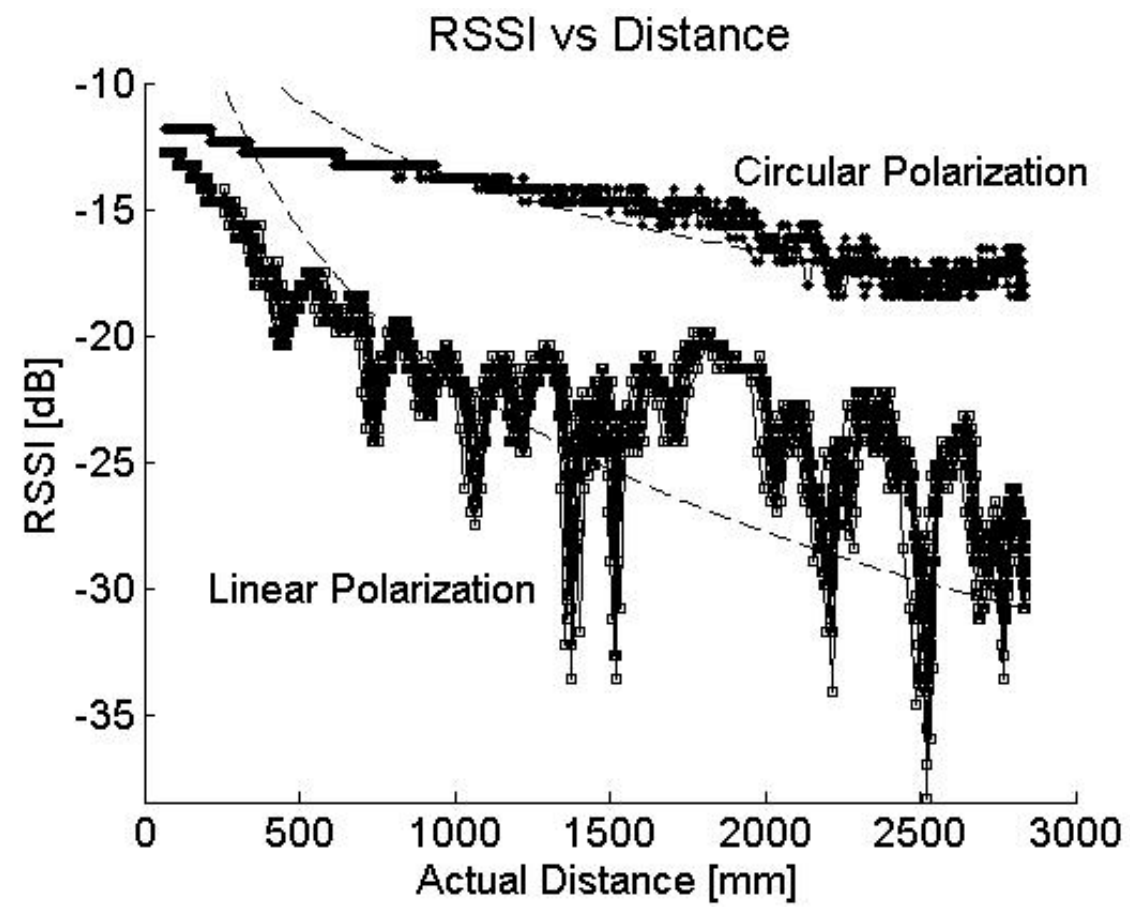

Fig. 3. RSSI as a function of the distance between the mobile node and an anchor, when linearly polarized (LP) or circularly polarized (CP) antennas are used (10 traces for each polarization case). Dashed lines are those obtained by a Hata-like model (see Eq. 1), when $\mathrm{d}_{0}=1 \mathrm{~m}$ and $\mathrm{n}_{\mathrm{p}}$ is equal to 1.99 and 0.98 , for LP and CP respectively.

The results in Fig. 3 demonstrate that $\mathrm{CP}$ could be a useful technique to get a smooth and predictable behaviour of the function RSSI-distance in indoor environments, as required for accurate localization purposes.

As a first tentative to show the effectiveness of CP in improving location accuracy, a standard Hata-like model [10] was implemented to relate measured RSSI and MNanchor distance:

$$
\operatorname{RSSI}_{(\mathrm{d}) \mathrm{dB}}=\operatorname{RSSI}_{(0) \mathrm{dB}}-10 \cdot \mathrm{n}_{\mathrm{p}} \cdot \log \left(\frac{\mathrm{d}}{\mathrm{d}_{0}}\right),
$$

where $n_{p}$ is the path loss coefficient (equal to 2 in ideal free-space conditions) and $d_{0}$ is a reference distance. For a set of values of the reference distance $d_{0}$ (between $25 \mathrm{~cm}$ and $2.5 \mathrm{~m}), \mathrm{n}_{\mathrm{p}}$ has been chosen to achieve the best fitting between the RSSI Hata-like model and the RSSI measurements. Experimental trials were repeated 10 times using two different antennas configurations. 
Localization performance was evaluated by computing the Root Mean Square Error (RMSE) between the estimated distance (using the Hata model) and the actual distance measured by the Vicon system. Numerical results are shown in Table 1 . In all experimental trials, $\mathrm{n}_{\mathrm{p}}$ values for LP case are always higher than those for the CP case. Minimum localization error $(\mathrm{RMSE}=40,4 \mathrm{~cm})$ is achieved within the CP set-up, by using $\mathrm{d}_{0}=1 \mathrm{~m}$ and $\mathrm{n}_{\mathrm{p}}=0,98$. LP shows worst localization performance because of the higher RSSI spatial fluctuations (see Fig. 3).

Further measurements have been planned, where the comparison between LP and $\mathrm{CP}$ antennas will be performed by using antennas with comparable beamwidths in both principal radiation planes.

Table 1. Location performance in terms of the Root Mean Square Error, RMSE, when linearly polarized (LP) or circularly polarized (CP) antennas are used

\begin{tabular}{|c|c|c|c|c|c|}
\hline \multirow{2}{*}{$\begin{array}{c}\text { Experimental } \\
\text { setup }\end{array}$} & \multicolumn{2}{|c|}{ LP } & \multicolumn{2}{|c|}{ CP } \\
\cline { 2 - 3 } \cline { 5 - 5 } & $\mathrm{n}_{\mathrm{p}}$ & $\begin{array}{c}\text { RMSE } \\
{[\mathrm{cm}]}\end{array}$ & & $\mathrm{n}_{\mathrm{p}}$ & $\begin{array}{c}\text { RMSE } \\
{[\mathrm{cm}]}\end{array}$ \\
\hline $\mathbf{d}_{\mathbf{0}}=\mathbf{2 5} \mathbf{c m}$ & 1,46 & 77,7 & & 0,51 & 65,4 \\
\hline $\mathbf{d}_{\mathbf{0}}=\mathbf{1 0 0 c m}$ & 1,99 & 64,5 & & 0,98 & 40,4 \\
\hline $\mathbf{d}_{\mathbf{0}}=\mathbf{1 2 5} \mathbf{c m}$ & 2,49 & 57,6 & & 1,09 & 42,6 \\
\hline $\mathbf{d}_{\mathbf{0}}=\mathbf{1 5 0} \mathbf{c m}$ & 3,13 & 62,9 & & 1,13 & 46,7 \\
\hline $\mathbf{d}_{\mathbf{0}}=\mathbf{1 7 5} \mathbf{c m}$ & 3,98 & 77,5 & & 1,33 & 60,9 \\
\hline $\mathbf{d}_{\mathbf{0}}=\mathbf{2 0 0} \mathbf{c m}$ & 3,34 & 78,7 & & 0,96 & 43,7 \\
\hline $\mathbf{d}_{\mathbf{0}}=\mathbf{2 2 5} \mathbf{c m}$ & 2,69 & 72,5 & & 1,05 & 45,4 \\
\hline $\mathbf{d}_{\mathbf{0}}=\mathbf{2 5 0} \mathbf{c m}$ & 2,90 & 59,5 & & 0,97 & 41,2 \\
\hline
\end{tabular}

\section{Conclusions}

An experimental activity on RSSI measurements in a $2.4 \mathrm{GHz}$ ZigBee-based wireless sensor network has shown that circularly polarized antennas (at both the mobile node and the anchors) can significantly reduce the fading in rich multipath indoor environments. By resorting to a conventional Hata-like model, it has also been shown that the resulting smooth behaviour of the RSSI as a function of the node-distance can guarantee for a better location accuracy when compared to that achievable in the same environment by using linearly polarized antennas. For the measurement set that we considered (linear path with a minimum distance equal to $25 \mathrm{~cm}$ and maximum distance equal to $250 \mathrm{~cm}$ ), we verified a localization error reduction, going approximately from $60 \mathrm{~cm}$ to $40 \mathrm{~cm}$, when switching from LP to CP. Better location accuracy can be obtained by resorting to advanced RSSI models and increasing the number of anchors, as well as by using data from the accelerometers already available in many commercial radio modules. However, for any network configuration that can be adopted, better performance is expected when $\mathrm{CP}$ antennas are used, due to the strong fading reduction that can be achieved.

Future work will be relevant to the extension of this research activity to more complex indoor scenarios and its application to Ambient Assisted Living systems. 


\section{Acknowledgment}

This work was developed within the ASTROMOBILE experiment project, courteously supported and funded by the ECHORD project.

\section{References}

1. Manabe, T., Sato, K., Masuzawa, H., Taira, K., Ihara, T., Kasashima, Y., Yamaki, K.: Polarization dependence of multipath propagation and high-speed transmission characteristics of indoor millimeter-wave channel at $60 \mathrm{GHz}$. IEEE Trans. on Vehicular Technology 44(2), 268-274 (1995)

2. Rappaport, T.S., Hawbaker, D.A.: Wide-band microwave propagation parameters using circular and linear polarized antennas for indoor wireless channels. IEEE Trans. on Communication 40(2), 240-245 (1992)

3. Huang, X.: Antenna polarization as a complementarities on RSSI Based Location Identification. In: ISWPC 2009, 4th International Symposium on Wireless Pervasive Computing (2009)

4. Potortì, F., Corucci, A., Nepa, P., Furfari, F., Barsocchi, P., Buffi, A.: Accuracy limits of in-room localisation using RSSI. In: IEEE Antennas and Propagation Society International Symposium (APS), Charleston, SC, US (2009)

5. Sathyan, T., Humphrey, D., Hedley, M., Johnson, M.: A wireless indoor localization network-system introduction and trial results. In: International global navigation satellite system society IGNSS Symposium (2009)

6. Bulusu, N., Heidemann, J., Estrin, D.: GPS-Less low cost outdoor localization for very small devices. IEEE Personal Communication Magazine, 7(5), 28-34 (2000)

7. Seco, F., Jimenez, A.R., Prieto, C., Roa, J., Koutsou, K.: A survey of mathematical methods for indoor localization. In: WISP 2009; 6th IEEE Internactional Symposiun on Intelligent Signal Processing (2009)

8. Gezici, S.: A survey on wireless position estimation. Wireless Personal Communication 44(3), 263-282 (2009)

9. Cavallo, F., Aquilano, M., Bonaccorsi, M., Carrozza, M.C., Dario, P.: Preliminary characterization of an indoor localization system using a ZigBee based sensor network and a triaxial accelerometer. In: AALIANCE Conference, Malaga, Spain, pp. 11-12 (2010)

10. Lymberopoulos, D., Lindsey, Q., Savvides, A.: An Empirical Characterization of Radio Signal Strength Variability in 3-D IEEE 802.15.4 Networks Using Monopole Antennas. In: Romer, K., Karl, H., Mattern, F. (eds.) EWSN 2006. LNCS, vol. 3868, pp. 326-341. Springer, Heidelberg (2006) 